\title{
LAGOCHILASCARIS MINOR LEIPER, 1909, (NEMATODA-ASCARIDAE) DE ORÍGEM HUMANA
}

\author{
Helio Martins de Araújo Cosxa (1). Amalia Verônica Mendes da SILva (2), Petrônio Rabelo \\ COSTA (3) \& Sandra Breder ASSIS (4)
}

\begin{abstract}
R E S U M O
Os autores isolaram e estudaram morfologicamente Lagochilascaris minor Leiper 1909, material obtido na cirurgia de um abscesso cervical direito de um menino de cor branca, com cinco anos de idade, procedente da cidade de Colorado, no Municipio de Vilhena, em Rondonia-Brasil. E sugerida uma chave para o diagnóstico diferencial das espécies do gênero Lagochilascaris.
\end{abstract}

UNITERMOS - Lagochilascaris minor -- Abscesso cervical em criança.

\section{N T R O D U G $\tilde{A} O$}

Atualmente são conhecidas cinco espécies do gênero Lagochilascaris: L. minor Leiper, 1909, L. major Leiper, 1910, L. turgida (Stossich, 1902) Travassos, 1924, L. buckleyi Sprent, 1971 e L. sprenti descrito por BOWMAN \& SMITH ${ }^{14}$; as três primeiras incluídas na revisão de SPRENT 15 ,

As espécies L. major e L. buckley foram descritas como parasitas de felídeos; L. turgida e L. sprenti, como parasitas de marsupiais. Enquanto isto, permanece desconhecido o hospedeiro de L. minor. A descrição original deste parasito foi baseada em material de origem humana.

A casuistica de infecções humanas por $\mathbf{L}$. minor continua crescendo, sobretudo com os achados brasileiros. Paralelamente surgem, também, referências de achados em outros hospedeiros.

O primeiro caso brasileiro de infecção humana por L. minor foi descrito por ARTIGAS \& col. 1. Posteriormente vieram os achados de
CORREA \& col. 8, BORGO \& col. ${ }^{3}$, LEÃO \& col. 11 , CHIEFFI \& col. 7, SANTOS \& col.14, BARBOSA CAMPOS \& col. ${ }^{2}$, MORAES \& col. ${ }^{12}$, FRAHIA \& col. 9 , CAMPOS \& col. ${ }^{6}$, LEÄO \& col. ${ }^{10}$, e OBEID \& col. ${ }^{13}$. Somando este caso aos registros anteriores passamos a 18 casos brasileiros. A literatura brasileira registrou também, com VIDOTTO \& col. ${ }^{16}$, Lagochilascaris minor em cão.

\section{MATERIAL E METODOS}

Em abril de 1980 chegou ao Hospital das Clínicas da Universidade Federal de Minas Gerais um menino, de cor branca, com cinco anos, procedente da cidade de Colorado, Municipio de Vilhena (Rondônia), o qual, desde os três anos de idade, apresentava dores de ouvido com corrimento purulento, melhorando sempre que fazia uso de antibiótico. Posteriormente desenvolveu uma intumescência na região cervical direita. a qual cresceu e se transformou em abscesso com fístula na parte inferior, com destruição do mastóide.

(1) Prof, do Dept. de Parasitologia ICB-UFMG. Bolsista do CNPa.

(2) Estudante de Pós-Graduação - Bolsista da CAPES.

(3) Prof. da Pediatria da Fac. de Medicina UFMG.

(4) Residiente do Hospital das Clinicas da UFMG. 
COSTA, F. M. de A.; SILVA, A. V. M. da; COSTA, P. R. \& ASSIS, S. B. - Lagochilascaris minor Leiper, 1909, (Nematoda-Ascaridae) de origem humana. Rev. Inst. Med. trop. Sào Paulo, 28:126-130, 1986.

Através desta fístula notou-se a eliminação de pequenos vermes. Já havia sido registrada a eliminação, também, pelo ouvido e narinas (espirro). A cirurgia do abscesso possibilitou a colheita de grande número de vermes, cerca de 200 , alguns dos quais foram colocados em placa de ágar-sangue humano e em tubos com ágar-sangue inclinado mais soro humano. Os outros foram fixados quente em soluções de Henry-Raillet.

Do abcesso foi colhido, também, material purulento para a pesquisa de ovos.

\section{RESULTADOS E DISCUSSAO}

Os helmintos colocados nas placas e nos tubos de ágar sangue não sobreviveram.

Os exemplares colhidos eram, na quase totalidade, vermes jovens.

Foram estudados 12 exemplares adultos, seis machos e seis fêmeas, com as caracteristicas abaixo:

Ascaridídeos com lábios apresentando profunda escavação mediana e separados do resto do corpo por nitida ranhura, delimitada inferiormente por um espessamento cuticular, do qual se originam os interlábios (Fig. $1 \mathrm{~A}, \mathrm{~B}, \mathrm{e} \mathrm{C}$ ). Labio dorsal com duas duplas papilas subdorsais; lábios sub-ventrais, cada um, com uma dupla papila ventro-lateral, uma papila lateral e o poro anfidial. Revestimento articular transversalmente estriado. Duas asas, pouco desenvolvidas, percorrem lateralmente 0 corpo e, praticamente, desaparecem no terço final. Esôfago simples; papilas cervicais inconspícuas, imediatamente após o anel nervoso, e quase que à mesma altura do poro excretor.

Machos - Cinco dos exemplares estudados mediram cêrca de 6 a $9 \mathrm{~mm}$ de comprimento e um era excepcionalmente grande, alcançando $14 \mathrm{~mm}$, inclusive maior dos que as fêmeas. Helmintos de pequeno porte, robustos e com a extremidade distal ligeiramente recurvada. Cauda curta, aparecendo em muitos exemplares uma protuberância terminal arredondada. Es. piculos fortes, praticamente do mesmo tamanho e dotados de ampla membrana alar (Fig. $1 \mathrm{E}$ e G). Duto ejaculador fortemente desenvolvido, atingindo cerca de duas a três vezes - tamanho do espículo. (Fig. 1 E). Papilas pós-cloacais variáveis em número e em disposição, (cinco a seis pares): uma papila dupla, de cada lado, imeđiatamente após a cloaca; duas papilas sub-ventrais e duas a três sub-dorsais (Fig. 1 D, E e G). Fasmides facilmente observadas em alguns machos. Ventral e imediatamente anterior à cloaca observa-se um par de papilas. As papilas pré-cloacais são numerosas, variando de 22 a 36 pares dispostos em duas fileiras laterais. Os seis a oito primeiros pares imediatamente anteriores à cloa. ca, são pedunculados (Fig. $1 \mathrm{E}$ ) são vistas na Tabela I.

TA B E L A I

Lagochilascaris minor - medidas em $\mathrm{mm}$

\begin{tabular}{|c|c|c|c|c|}
\hline & \multicolumn{2}{|c|}{ Machos } & \multicolumn{2}{|c|}{ Fêmeas } \\
\hline & Média & Amplitude & Média & Amplitude \\
\hline Comprimento do corpo & 8,7 & $5,9-14,0$ & 9,0 & $7,4-10,5$ \\
\hline Largura máxima & 0,437 & $0,271-0,650$ & 0,514 & $0,406-0,558$ \\
\hline Largura na cabeça & 0,144 & $0,088 \cdot 0,175$ & 0.166 & $0,147-0,197$ \\
\hline Largura na cloaca ou fanus & 0,148 & $0,134 \cdot 0,184$ & 0,175 & $0,152-0,200$ \\
\hline Comprimento do esofago & 1,000 & $0,731-1,500$ & 1,100 & $1,000-1,300$ \\
\hline Dist. anel nervoso à extrem. anterior & 0,320 & $0,238-0,406$ & 0,335 & $0,270 \cdot 0,434$ \\
\hline Dist, poro excretor à extrem. anterior & 0,377 & $0,314 \cdot 0,461$ & 0,403 & $0,352-0,531$ \\
\hline Altura do lábio & 0,054 & $0,038-0,066$ & 0,060 & $0,035-0,077$ \\
\hline Comprimento da cauda & 0,109 & $0,073 \cdot 0,141$ & 0,135 & $0,108-0,173$ \\
\hline Dist. vulva à extrem. anterior & - & - & 4,590 & $3,710-5,310$ \\
\hline Relaçāo dist. vulva: comp. corpo & - & - & $1: 1,97(50,8 \%)$ & $\begin{array}{l}1: 1,878(53,25 \%) \\
1: 1,895(50,13 \%)\end{array}$ \\
\hline Largura do corpo na vulya & - & - & 0,483 & $0,390 \cdot 0,547$ \\
\hline Comprimento dos espiculos & 0,359 & $0,266-\bigcap, 699$ & - & - \\
\hline Comp. do duto ejaculador & 0,780 & $0,569 \cdot 1,257$ & - & - \\
\hline Relação espículo: duto ejaculador & $1: 2,17(46,0 \%)$ & $\begin{array}{l}1: 1,80(55,61 \%)- \\
1: 2,95(33,92 \%)\end{array}$ & - & - \\
\hline Ovos no útero da femes & - & - & $0,051 \times 0,069$ & $0,042 \cdot 0,066 \times 0,058 \cdot 0,086$ \\
\hline Oyos na secreçắ purulenta & - & - & $0,066 \times 0,079$ & $0,058-0,074 \times 0,064-0,098$ \\
\hline
\end{tabular}


CostA, H. M. de A.; SILvA, A. V. M. da; COSTA, P. R. \& AssiS; S. B. - LAgochllsscarts miner Ielper, 1909, Gema toda-Ascaridae) de origern humana. Rev. Inst. Med. trop. Säo Paulo, 28.tas-130, 1966.

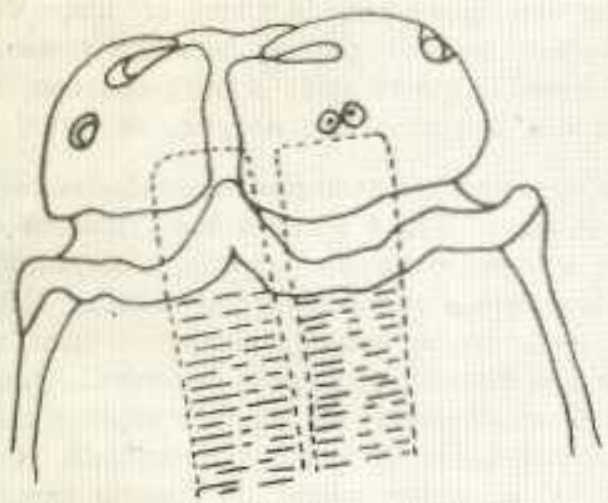

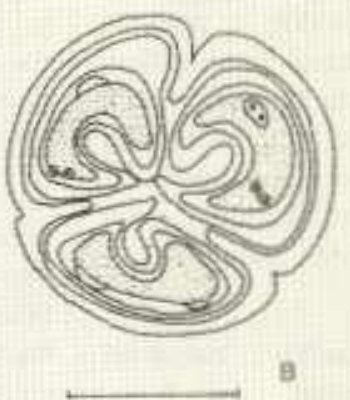

Q $\mathrm{mm}$
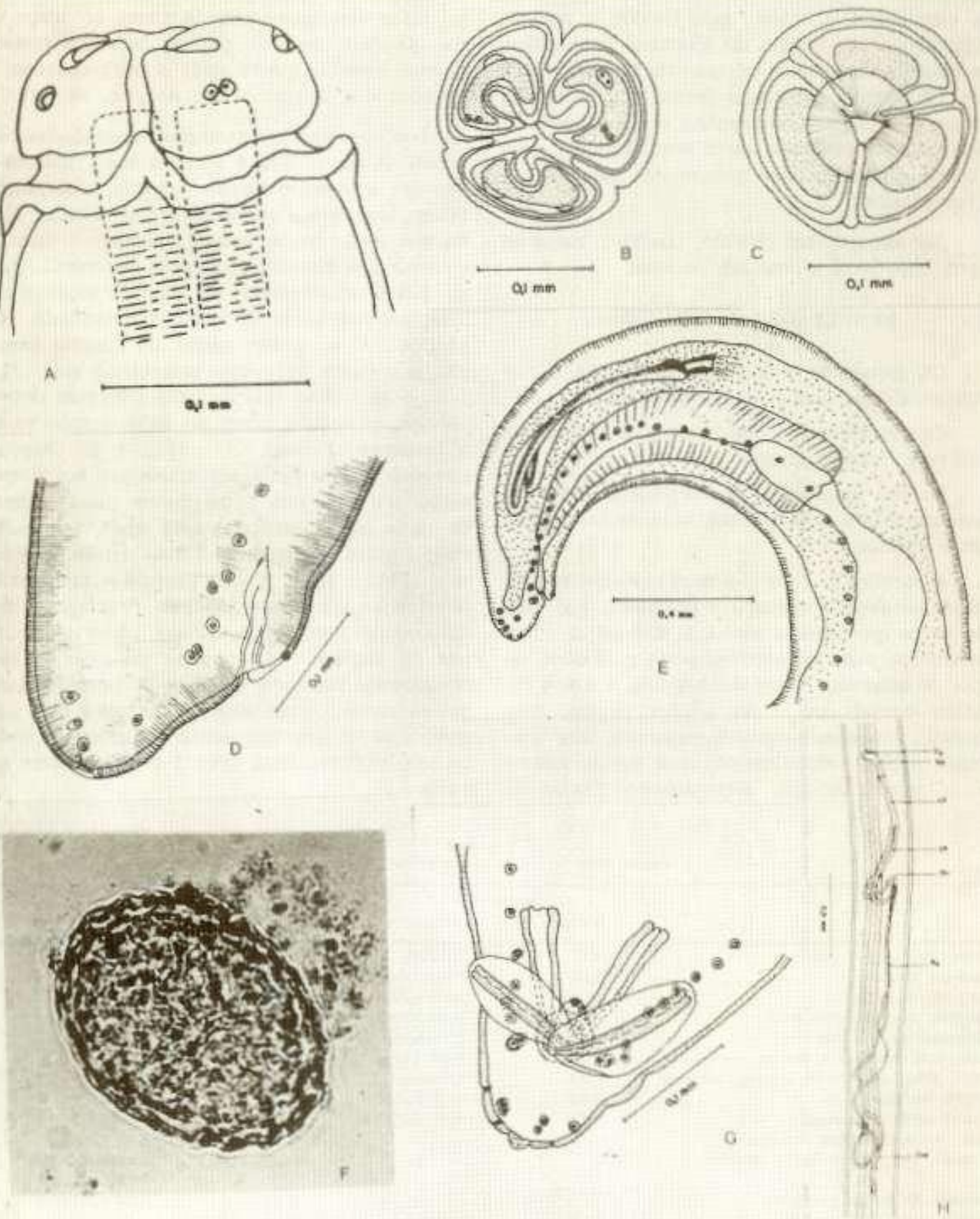

Fig. 1 - Lagochilascaris minor: A) Extremidade cefálica. B) e c) - Vista frontal da extremidade cefalica. D) - Ex tremidade caudal do macho em viata lateral. E) - Extremidade caudal do macho em vista lateral, apurecendo os espiculos o duto ejaculador e as papilas. F) - Ovo colhido com a secreçlo purutenta durante a eirurgia. G) - Extremidade cautal do macho (vista ventral), aparecendo os espleulos e alrumas papilas $\mathrm{H}$ ) - Pémea jovem: Vu $=\mathrm{Vulva}$, Va $=$ vagina $\mathrm{Ut}=$ utero, $\mathrm{Ov1}=$ oviduto: $\mathrm{Rs}=$ teceptaculo seminal $e$ ovo $=$ ovirio 
COSTA, H. M. de A.; SILVA, A. V. M. da; COSTA, P. R. \& ASSIS, S. B. - Lagochllascaris minor Leiper, 1909, (Nemª. toda-Ascaridae) do origem humana, Rev. Inst. Med. trop. São Paulo, 28:126-130, 1986.

Fêmeas - Dimensó̀es na Tabela I. Mediam cerca de 7,5 a $10,5 \mathrm{~mm}$ de comprimento, ligeiramente maiores que os machos e apresentavam ovos no útero. Cauda curta e reta geralmente desprovida de tubérculo terminal. Abertura vulvar simples e situada imediatamente após a metade anterior do corpo; esta distância da extremidade anterior variando de 50,13 a $53,25 \%$ do comprimento total do corpo. Prodelfas, com vagina relativamente longa $e$, nas fêmeas adultas, recurvada para trás, comunicando-se com o útero, que é único em sua porção anterior e depois se divide em dois ramos, sujas porçōes terminais se comunicam com os ovidutos. Alças uterinas situadas na região pós-vaginal, algumas vezes projetando-se um pouco anteriormente à vagina. Fêmeas adultas com a porção única e os dois ramos do útero repletos de ovos. Ovos de contorno arre dondado e ovalado, de casca espessa e irregular, apresentando menos de 25 depressões em torno da linha equatorial (Fig. $1 \mathrm{~F}$ ); dimensóes variáveis, inclusive com a fêmea de orígem. Medidas de alguns ainda no interior do utero mostram que variam de 42 a $66 x$ a 58 a $86 \mu$. Ovos eliminados, com o material purulento do abscasso, pouco numerosos, mediam de 40 a $83 \times 58$ a $98 \mu$; um ovo arredondado chegou a medir $83 \mu$ de diâmetro; entre os ovalados o menor media $40 \times 52 \mu$ e o maior $73 \times 98 \mu$. Material purulento lavado em água, centrifugado a $2.000 \mathrm{rpm}$ durante dois minutos e ressuspenso em água, conservado em geladeira, apresentou ovos larvados 30 dias após.

O diagnóstico diferencial de L. minor com L. turgida, L. buckleyi, L. sprenti é fácil. Morfologicamente L. minor e L. major muito se assemelham, como se pode ver na literatura existente, inclusive em SPRENT ${ }^{15}$, O número de depressões em torno da linha equatorial do ovo (L. minor $=15-25$ e L. major $=35-45$ ) é considerado o único elemento para a separação, o que nos parece muito pouco.

O apêndice caudal arredondado, assinalado em alguns machos de $\mathbf{L}$. major, pode ser visto também em L. minor.

O grande número e a variedade de formas evolutivas encontradas no abscesso sugerem a possibilidade de, a partir da infecção inicial, ocorrer multiplicação no próprio hospedeiro, hipótese já levantada por BRUIJNING ${ }^{5}$ e MORAES \& col. 2 .

\section{CONCLUSOEES}

Face aos dados apresentados na literatura existente, os Autores sugerem o diagnóstico diferencial das espécies do gênero Lagochilascaris como segue:

\section{CHAVE PARA O DIAGNÓSTICO DAS ESPE- CIES DO GENERO LAGOCHILASCARIS}

1. Espículos de tamanho igual ou maior do que o duto ejaculador; vilva anterior go meio do corpo, cerca de $3245 \%$ do comprimento do mesmo:

- Espiculos de tamanho igual ou ligeiramente maior do que o duto ejaculador; interlabios em forma de lança $\ldots \ldots \ldots \ldots \ldots$ turgida.

- Espículos medindo cerca de duas vezes mais do que o duto ejaculador $\ldots \ldots \ldots \ldots \ldots \ldots \ldots \ldots$ L. buckleyi.

2. Espículos ligeiramente menores ou bem menores do que o duto ejaculador:

- Comprimento do espículos de 0,75 a $1,19 \mathrm{~mm}$, correspondendo a cerca de $83 \%$ $(59,5-99,8)$ do comprimento do duto ejaculador; ovos com 24 a 31 depressōes na linha equatorial; vulva anterior ao meio do corpo, a uma distância de 32 a $48 \%$ do comprimento do mesmo. . . . . . . . . . . . . . . . . L. sprenti.

- Espículos medindo $0,475-0,925 \mathrm{~mm}$, mais curtos do que o duto ejaculador e equívalentes a $59,4-84.1 \%$ do mesmo; vulva na metade posterior do corpo, a cerca de $51.56 \%$ do comprimento; ovos com 35-45 depressoes na linha equatorial . . . . . . . . . . . . . . . L. major.

- Espículos medindo de 0,23-0,70 $\mathrm{mm}$, bem menores do que o duto ejaculador, equivalentes a $3456 \%$ do comprimento do mesmo, vulva geralmente posterior ao meio do corpo, situada de $47-60 \%$ do comprimento do corpo; ovos com 15-25 depressões na linha equatorial.

. . . . . . . . . . . . . . . . . L. minor.

\section{SUMIMARY}

Lagochilascaris minor Leiper, 1909 (NematodaAscarididae) from human origin 
COSTA, H. M. de A.; SILVA, A. V. M. da; COSTA, P. R. \& ASSIS, S. B. - Lagochilascaris minor Leiper, 1909, (Nema. toda-Ascaridae) de origem humana. Rev. Inst. Med. trop. São Paulo, 28:126-130. 1986.

The Authors studied parasites obtained by surgery in the right cervical region of a child from the city of Colorado, county of Vilhena, in the State of Rondonia-Brazil. This is 18th Brazilian case described. The Authors suggest a key to the known species of Lagochilascaris.

\section{AGRADECIMENTOS}

Agradecemos ao Prof. Petrônio Rabelo Costa e à Residente Sandra Breder Assis do Hos pital das Clínicas da UFMG por nos haverem encaminhado o material para o diagnóstico.

\section{REFERENCIAS BIBLIOGRAFICAS}

1. ARTIGAS, P. T.; ARAUJJO, P.; ROMITI, N. \& RUIVO, M. - Sobre um caso de parasitismo humano por La. gochilascaris minor Leiper, 1909, no Estado de Săo Paulo. Rev. Inst. Med. trop. S. Paulo, 10: 78.83, 1968.

2. BARBOSA CAMPOS, D. M.; DOBLER KOMMA, M.; QUEIROZ SANTOS, M. A. \& VASCONCELOS PINTA LUNA, W. M. N. - Lagochilascaris minor Leiper, 1909: casos diagnosticados no Departamento de Parasitologia. In: CONGRESSO DA FEDERACION LATINOAMERICANA DE PARASITOLOGOS, 6.; CONGRESSO DÁ SO. CIEDADE BRASTLEIRA DE PARASITOLOGIA, 8. \& JORNADA PAULISTA DE PARASITOLOGIA, 5., São Paulo, 1983. Resumos. São Paulo, FLAP, 1983. p. 100. (Congressos Integrados de Parasitologia, 1983).

3. BORGO, A. V.; ANDRADE, A. L. S.; PEDROSA, R. B.; BARBOSA, W. \& KOMMA, M. D. - Infecçāo por Lagochilascaris minor. Apresentaçāo de caso. In: CONGRESSO DA SOCIEDADE BRASILEIRA DE ME. DICINA TROPICAL, 15. \& CONGRESSO DA SOCIEDA. DE BRASILEIRA DE PARASITOLOGIA, 3., Joāo Pessoa, 1978. Resumos dos temas-livres. João Pessoa, Eda. Universitária da UFPb, 1978, p. $39 t$.

4. BOWMAN, D. D.; SMITH, J. L. \& LITTLE, M. D. Lagochliascaris sprenti sp. $n$. (Nematoda: Ascarididae) from the opossum Didelphis virginiana (Marsupialia: Didelphidae). J. Parasit., 69: 754-760, 1983.

5. BRUIJNING, C. F. A. - Note on Lagochilascaris minor Leiper, 1909. Docum. Med. Geog. Trop., 9: 173.175, 1957. APUD: MORAES \& col., 1983.

6. CAMPOS, R.; VIEIRA BRESSAN, M. C. R.; LITTLE, M. D.; ROSEMBERG, S. ; PEREIRA, V. C. \& MASUDA, z. - Encefalopatia aguda por Lagochilascaris minor Leiper, 1909. II Aspectos parasitológicos. In: CONGRESSO DA. SOCIEDADE BRASILEIRA DE MEDICINA TROPICAL, 21., São Paulo, 1985. Programa e resumos. Sāo Raulo, Balieiro, 1985. p. 74.

7. CHIEFFI, P. P.; PASCHOALOTTI, M. A.; FRUCCHI, H. ; PER̨EIRA, W. A. \& PROENÇA, N. G. - Infecçāo por Lagochilascaris minor (Leipper, 1909), uma helmin. tíase rara no ser humano. In: CONGRESSO BRASI-
LEIRO DE PARASITOLOGIA, 5., Río de Janeiro, 1980. Resumos. Rio de Janeiro, FIOCRUZ, 1980. p. 95.

8. CORREA, M. O. A.; HYAAKUTAKE, S.; BRANDI, A. I. \& MONTEIRO, C. G. - Novo caso de parasitismo humano por Lagochilascarls minor Leiper, 1909. Rev. Inst. A. Lutz (S. Paulo), 38: 59.65, 1978.

9. FRAHIA, H.; ROCHA, M. P. C.; ARAUJO, O. J.; BARROS, V. L. R. S.; PRIMO, A.; MORAES,. M. A. P.; CONCEIÇAO, J. R. \& OLIVEIRA, J. E. G. Patologia amazónica. II. Infecçāo humana por Lago. chilascaris Leiper, 1909 (Nematoda-Ascarididae), Registro de três novos casos e formulaçāo de nova hipotese para o mecanismo do infecção. In: CONGREsSO DA FEDERACION LATINOAMERICANA DE PARASITOLOGOS, 6.; CONGRESSO DA SOCIEDADE BRASI LEIRA DE PARASITOLOGIA, 8. \& JORNADA PAULISTA DE PARASITOLOGIA, 5., Sāo Paulo, 1983. Resumos. Săo Paulo, FLAP, 1983. p. 146. (Congressos Integrados de Parasitoiogia, 1983).

10. LEAO, R. N. Q.; FRAHIA NETO, H.; FRAHIA, S. C.: TONINL, K. C. \& SILVA, J. A. P. R. - Perspectivas de emprego do Cambendazol na lagoquilascarfase. In: CONGRESSO DA SOCIEDADE BRASILEIRA DE MEDICINA TROPICAL, 21., São Paulo, 1985, Programa e resumos. São Paulo, Balieiro, 1985. p. 76.

11. LEAO, R. N. Q.; LEAO FILHO, J.; DIAS, L. B. \& CALHEIROS, L. B. - Infecção humana pelo Lagochi. lascaris minor Leiper, 1909: registro de um caso obser. vado no Estado do Pará (Brasil). Rev. Inst. Med trop. S. Paulo, 20: $300-306,1978$.

12. MORAES, M. A. P.; ARAUD, M. V. C. \& LIMA; P. L - Novos casos de infecção humana por Lagochilascaris minor Leiper, 1909, encontrados no Estado do Pará, Brasil, Rev. Inst. Med, trop. S. Paulo, 25: 139.146, 1983.

13. OBEID, J. N.; FRAHIA NETO, H.; VIEIRA, F. P. \& ABREU, E. P. - Lagoquilascariase com envolvimento cerebelar. In: CONGRESSO DA SOCIEDADE BRASILEIRA DE MEDICINA TROPICAL, 21., São: Paulo, 1985. Programa e resumos. São Paulo, Balieiro, 1985 p. 80 .

14. SANTOS, M. A. Q.; CAMPOS, D. M. B. \& BARNABP, W. - Lagochilascaris minor Leiper, 1909, em abcesso dentário, no Estado de Goiás. In: CONGRESSO BRA. SILEIRO DE PARASITOLOGIA, 6., Belo Horizonte, 1981. Resumos. Belo Horizonte, Imprensa Universitária da UFMG, 1981. p. 131.

15. SPRENT, G. F. A. - Speciation and development in genus Lagochilascaris. Parasitology, 62: 71-112, 1971.

16. VIDOTTO, O.; ARAÛJO, R.; ARTIGAS, P. T.; REIS, A. C. F.; VIOTTI, N. M. A.; PEREIRA, E. C. P. \& YAMAMURA, N. H. - Caso de lagoquilascariose minor em cāo. In: CONGRESSO BRASILEIRO DE PARASI TOLOGIA, 7., Porto Alegre, 1982 . Resumos. Porto Ale gre, Sociedade Brasileira de Parasitologia, 1982. p. 76.

Recebido para publicaçăo em $18 / 3 / 1985$. 